\title{
Aspects épidémiologiques, cliniques et thérapeutiques des gangrènes de Fournier dans le service d'urologie du CHU de Point-G à Bamako (Mali)
}

\section{Therapeutic, clinical, and epidemiological aspects of Fournier's Gangrenes in the Urology Department at Point-G University Hospital in Bamako (Mali)}

\author{
1. Service d'Urologie CHU Point G \\ 2. Service d'Urologie CHU BSS de Kati \\ 3. Service d'Urologie CHU Gabriel Touré \\ 4. Service d'Urologie Hôpital Sominé Dolo de Mopti \\ 5. Service d'Urologie CHU de Luxembourg
}

Diallo $\mathrm{MS}^{2}$, Coulibaly $\mathrm{MT}^{3}$, Cissé $\mathrm{D}^{4}$, Berthé $\mathrm{HJG}^{1}$, Kassogué $\mathrm{A}^{2}$, Diarra $\mathrm{A}^{5}$, Tembély $\mathrm{A}^{1}$

Auteur correspondant: Dr Diallo Moussa Salifou, Service d'urologie du centre hospitalouniversitaire Pr Bocar Sidy SALL de Kati Email: mousalifon@gmail.com

\begin{abstract}
Résumé
Objectif : Etudier les aspects épidémiologiques, cliniques et thérapeutiques des gangrènes de Fournier dans le service d'urologie du CHU du Point G à Bamako (Mali).Patients et Méthodes : Nous avons effectué une étude rétrospective transversale et descriptive de tous les patients admis pour Gangrène de Fournier et pris en charge dans le service d'urologie du C.H.U de Point G sur une période de quatre ans allant du $1^{\text {er } J a n v i e r ~} 2009$ au 31 Décembre 2012. Les paramètres étudiés étaient : l'âge, le sexe, le motif de consultation, les facteurs de risque, l'étendue des lésions, les méthodes thérapeutiques utilisées et le résultat des traitements. Résultats : La fréquence de la gangrène de Fournier représentait $1,2 \%$ des pathologies urologiques enregistrées. La moyenne d'âge était de 49 ans avec des extrêmes de 22 et 76 ans. Une cause urinaire était notée dans $26 \%$ des cas. Les germes rencontrés étaient Escherichia coli dans $26 \%$ des cas suivi de Klebsiella pneumonie et Staphylococcus aureus. La tuméfaction des organes génitaux externes a été l'expression clinique la plus fréquente dans $56 \%$ des cas. Les lésions avaient une étendue périnéo-scrotale dans $65 \%$ des cas. Les patients étaient traités par une triple antibiothérapie dans $87 \%$ des cas suivie d'une excision des tissus nécrotiques avec mise à plat et drainage urinaire. Conclusion: La gangrène de Fournier est une affection rare mais très grave. Une prise en charge médico-chirurgicale intensive et précoce améliore le pronostic de façon spectaculaire.
\end{abstract}

Mots clés: Antibiothérapie, Gangrène de Fournier, Nécrosectomie.

\section{Abstract}

Objective: To study the epidemiological, clinical and therapeutic aspects of Fournier's gangrenes in the Urology Department at Point-G University Hospital in Bamako (Mali).Patients and Methods: We performed a crosssectional and descriptive retrospective study of all patients admitted to Fournier's gangroen and managed in the urology department of the Point G CHU over a four-year period from January 1, 2009 to December 31, 2009. December 2012. The parameters studied were: age, sex, reason for consultation, risk factors, extent of lesions, therapeutic methods used and treatment outcome. Results: The frequency of Fournier gangrene was $1.2 \%$ of pathologies with an average age of 49 years with extremes of 22 and 76 years. A urinary cause was noted in $26 \%$ of cases. The most frequently encountered organisms were Escherichia coli in $26 \%$ followed by Klebsiella pneumonia and Staphylococcus aureus. Swelling of the external genital organs was the most common clinical expression in $56 \%$ of cases. Lesions had perineal-scrotal extent in $65 \%$ of cases. The patients were treated with triple antibiotic therapy in $87 \%$ of cases followed by excision of necrotic tissue with flattening and urinary drainage. The average duration of hospitalization was 65 days. Conclusion: Fournier's gangrene is a rare but very serious condition. Intensive medico-surgical management improves the prognosis dramatically.

Key words: Antibiotic therapy, Fournier's gangrene, Necrosectomy.

Introduction: La gangrène de Fournier (G.F) est une cellulo-fasciite grave nécrosante et rapidement progressive du périnée et des organes génitaux externes [1]. Elle est secondaire à une infection poly microbienne par des bactéries aérobies et anaérobies ayant une action synergétique [1]. La gravité particulière de cette pathologie nécessite ainsi un index de suspicion élevé, afin de procéder à un diagnostic précoce et de mettre en route très rapidement un traitement agressif médicochirurgical [1]. Elle peut engager le pronostic vital du patient avec une mortalité qui est autour de $20 \%$ [2]. Des facteurs de risque comme l'âge, le diabète et l'immunodépression, sont souvent présents chez les patients atteints. La base du traitement est une chirurgie d'exérèse rapide sur les tissus nécrosés associée à une antibiothérapie à large spectre. L'objectif de ce travail est d'étudier les aspects épidémiologiques, cliniques et thérapeutiques des gangrènes de Fournier dans le service d'urologie du CHU du Point G à Bamako (Mali). 
Patients et Méthodes: Il s'agissait d'une étude rétrospective transversale et descriptive de tous les patients admis pour GF et pris en charge dans le service d'urologie du CHU de Point G sur une période de quatre ans allant du $1^{\text {er }}$ Janvier 2009 au 31 Décembre 2012. Les paramètres étudiés étaient : l'âge, le motif de consultation, les facteurs de risque, l'étendue des lésions, les méthodes thérapeutiques utilisées et le résultat des traitements. Nos sources d'information étaient: les registres de consultation, les dossiers médicaux, les registres d'hospitalisation, les registres de compte rendu opératoire. Les données ont été saisies et analysées sur le logiciel Epi info version 6.0.

Résultats: Au cours de notre étude nous avons noté 23 cas de G F sur 1711 consultations soit 1,2\% des pathologies. L'âge moyen était de 49 ans avec des extrêmes de 22 et 76 ans (figure 1).

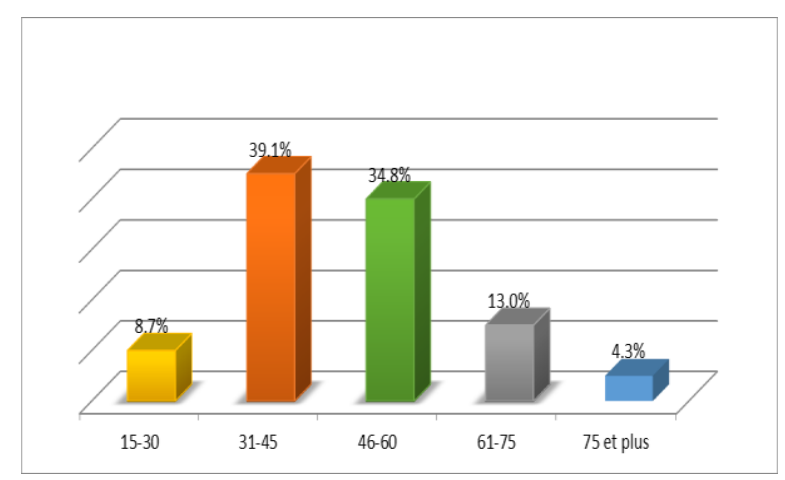

Figure 1: Répartition des patients selon la tranche d'âge

Un niveau économique bas a été enregistré dans 52 $\%$ des cas. Un terrain diabétique a été retrouvé chez $22 \%$ de cas avec une cause urinaire notée dans $26 \%$ des cas et $4 \%$ de cause ano-rectale. Le délai moyen de consultation a été de 11 jours. Le motif de consultation retrouvé était la tuméfaction des organes génitaux externes dans $56 \%$ suivie des douleurs périnéo-scrotales dans 13\% (Tableau 1).

Tableau I: Répartition des patients selon le motif

\begin{tabular}{lcc}
\multicolumn{3}{c}{ de consultation } \\
\hline Motif consultation & Fréquence & Pourcentage \% \\
\hline Douleur scrotale & 2 & 8,7 \\
Abcès périnéal & 2 & 8,7 \\
Tuméfaction & 12 & 52,2 \\
scrotale & & \\
Dysurie & 1 & 4,3 \\
Tuméfaction des & 1 & 4,3 \\
OGE & & \\
Douleur périnéale & 1 & 4,3 \\
Nécrose des bourses & 1 & 4,3 \\
Plaie scrotale & 3 & 13,2 \\
Total & $\mathbf{2 3}$ & $\mathbf{1 0 0 , 0}$ \\
\hline
\end{tabular}

Une étiologie uro-digestive a été retrouvée dans $52 \%$ des cas : abcès péri-urétral $26 \%$, traumatisme uro-génital $22 \%$ et l'abcès anal dans $4 \%$. Dí Gauche $\%$ des cas nous avons retrouvé des facteurs ae risque qui étaient le diabète dans $22 \%$ et le VIH dans $4 \%$ des cas. L'examen physique a retrouvé une odeur fétide caractéristique chez tous les patients suivie de la fièvre et la phlyctène dans $91 \%$ des cas pour chacune. Les lésions avaient une étendue périnéo-scrotale dans $65 \%$ des cas, scrotale $13 \%$ des cas (figure 2) et l'atteinte de l'abdomen était notée dans $9 \%$ des cas (Figure 3).

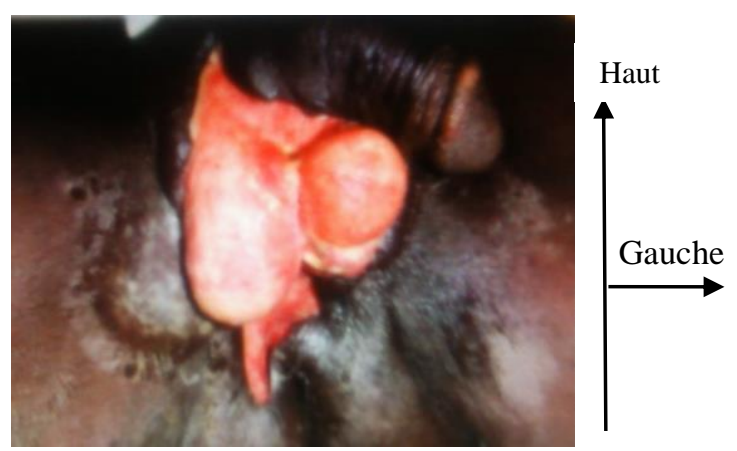

Figure 2: Lésions de gangrène scrotale en phase de restauration (image du service).

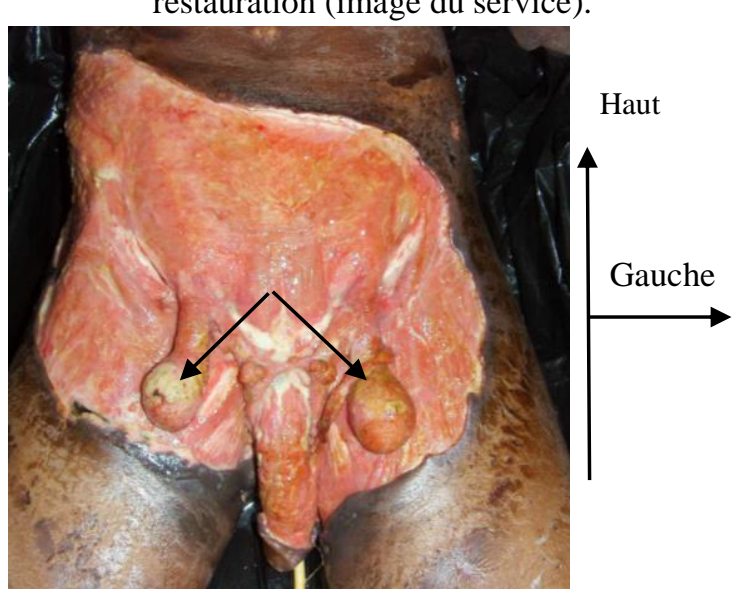

Figure 3: Gangrène des organes génitaux externes étendue à l'abdomen avec les testicules à nu (flèches) et la verge après une nécrosectomie large.

La culture bactériologique était positive dans $83 \%$ des cas avec E- coli dans $26 \%$, suivi de Klebsiella pneumoniae dans 13\%; le Staphylococcus aureus, Proteus mirabilis et Claustrodium perfringens ont représenté chacun $9 \%$ des cas et une culture poly microbienne était noté dans $13 \%$ (tableau 2).

Tableau II: Répartition des patients selon le résultat de la culture bactériologique

\begin{tabular}{lcc}
\hline Culture & Fréquence & Pourcentage \% \\
\hline E. coli & 6 & 26,0 \\
K. pneumonie & 3 & 13,0 \\
S. aureus & 2 & 8,7 \\
P. mirabilis & 2 & 8,7 \\
C. perfringens & 2 & 8,7 \\
Proteus + E. Coli & 1 & 4,3 \\
Streptocoque + E. & 1 & 4,3 \\
Coli & & \\
Streptocoque + & 1 & 4,3 \\
Staphylocoque & & \\
Non fait & 1 & 4,3 \\
Négatif & 4 & 17,3 \\
Total & $\mathbf{2 3}$ & $\mathbf{1 0 0 , 0}$ \\
\hline
\end{tabular}


La cystostomie de dérivation a été effectuée dans $74 \%$ des cas, un sondage transurétrale dans $22 \%$ des cas. L'antibiothérapie associée à une héparinothérapie ont été systématique chez tous nos patients. Cette antibiothérapie était triple dans $87 \%$ des cas. Une double antibiothérapie a été instaurée dans $8,7 \%$ des cas et 1 cas a reçu un traitement avec un seul antibiotique à base de Céftriaxone $2 \mathrm{~g}$ sur 24 heures pour insuffisance rénale. Cette antibiothérapie a été poursuivie jusqu'à 5 jours après apyrexie chez nos malades. Les gestes chirurgicaux ont été une nécrosectomie économe chez 16 patients (69\% des cas), une nécrosectomie large chez 5 patients (22\% des cas) et une mise à plat + drainage de l'abcès chez 2 patients $(9 \%)$. Après cette phase de nécrose et d'infection, une chirurgie de reconstruction a été effectuée qui consistait à un enfouissement des testicules $(n=14)$; un rapprochement scrotale $(n=4)$ et une greffe cutanée $(n=2)$. Le délai de cicatrisation variait entre 8 et 61 jours avec une durée moyenne de 27 jours. La durée d'hospitalisation était variable entre 10 et 143 jours avec une moyenne de 65 jours. Le suivi à court et moyen terme a permis de retrouver des cicatrices indélébiles chez 12 patients et 1 cas de déformation de la verge.

Discussion: La GF est une fasciite nécrosante infectieuse des régions génitale, périnéale ou périanale [1]. Elle a fait l'objet de plusieurs articles depuis sa première description en 1883, Jean Alfred Fournier [3]. Elle correspond à un état histopathologique caractérisé par l'infection aiguë du tissu cellulaire sous cutané par des germes anaérobies et/ou bacilles Gram négatif, évoluant de façon rapide et imprévisible à la nécrose et la gangrène des parties molles de l'aire génitale et périnéale [4]. Elle reste rare dans les pays développés, avec moins de 700 cas publiés sous forme de cas isolés ou de séries courtes [5]. Dans notre série la gangrène a représenté $1,2 \%$ de toutes les pathologies prises en charge pendant la période d'étude. Ces résultats sont similaires à ceux de Hodonou $\mathrm{R}$ et al. au Bénin qui ont trouvé 1,17\% de cas Gangrène de Fournier [6]. La GF est une pathologie du sujet âgé [4], mais elle peut se rencontrer à tous les âges. Dans notre série, nous avons trouvé une moyenne d'âge de 49 ans. La gangrène est une pathologie multifactorielle. L'infection des fascias périnéaux à la base de la gangrène peut avoir une origine soit locale ou à distance. Nous avons retrouvé une cause urodigestive dans $52 \%$ des cas dont les abcès périurétraux étaient le plus fréquents avec $26 \%$ de ces cas. Il existe des formes idiopathiques, ils représentaient $22 \%$ dans notre étude. Toutes les couches socio-économiques peuvent être concernées mais notre série a trouvé un niveau économique bas dans $52 \%$.
Les symptômes commencent par un œdème initialement localisé se généralisant rapidement à l'ensemble des OGE. Une sensation de brûlure et de striction, avec parfois des prurits l'accompagnent. Cette sensation se transforme très rapidement en douleur vive spontanée [5]. Ceci traduit le début d'une cellulite et qui va entraîner une fasciite nécrosante et des thromboses vasculaires. Au bout de quatre à cinq jours la gangrène est évidente, la douleur diminue par destruction nerveuse, l'emphysème sous cutané et les plages noirâtres de sphacèle apparaissent et dégagent une odeur fétide caractéristique de l'anaérobiose [7].La fièvre signe de l'infection et de l'inflammation était présente dans $91 \%$ associée à une phlyctène scrotale dans notre étude, Hodonou $\mathrm{R}$ et al ont trouvé 17 cas de fièvre soit 53\% [6].La gangrène de Fournier représente plusieurs entités anatomopathologiques; dans notre série les lésions étaient étendues au niveau périnéo-scrotale dans 65 $\%$ des cas pendant que Ettalbi S et al [8] et Borki K et al [4] ont trouvé des formes extensives avec atteinte de la paroi abdominale respectivement dans $44 \%$ et $54 \%$ des cas.Tous nos patients ont été vus à un stade tardif avec un délai moyen de consultation de 11 jours. Cela nous a permis de poser le diagnostic clinique de façon aisée. Certains auteurs [6,9] recommandent l'utilisation de l'imagerie (ultrasons, IRM et scanner) pour poser le diagnostic précoce. La culture a été positive dans $83 \%$ des cas. Elle a été mono-microbienne avec Ecoli dans $26 \%$ des cas et poly microbienne dans $13 \%$ des cas. Ces résultats sont différents de ceux de Toutti D et al [10] au Maroc qui ont trouvé une culture positive à E-coli dans $31 \%$ et poly microbienne dans la $34 \%$ des cas. L'antibiothérapie est de règle et doit être instauré de façon rapide et probaliste avant d'avoir le résultat de la culture. Nous avons instauré une triple antibiothérapie composée d'une $\beta$-lactamine (ceftriaxone 2grammes), un aminoside (Gentamycine 160mg) et un imidazolé (metronidazole $1,5 \mathrm{~g}$ ) avec succès dans $87 \%$ des cas. Cette même principe de triple antibiothérapie a été observé par certains auteurs $[4,11]$. La réanimation médicale a consisté à un équilibre hydroélectrolytique et une héparinothérapie dans tous les cas ; une sérothérapie antitétanique dans 19 cas. Le geste chirurgical de première intention a été une nécrosectomie économe dans $69 \%$ cas et une nécrosectomie large dans $22 \%$ cas. Ce même principe de nécrosectomie économe a été observé chez K Dje et al en Côte d'Ivoire dans 95\% pour une nécrosectomie élargie dans $5 \%$ [2]. $\mathrm{R}$ Hodonou et al ont effectué une bipartition scrotale et une nécrosectomie élargie dans $77 \%$ de leur série [6]. La dérivation urinaire par cystostomie a été utilisée dans $74 \%$ des cas et aucun drainage n'a été fait chez $4 \%$ de nos patients. La chirurgie de reconstruction a consisté à un enfouissement des 
testicules dans 14 cas; un rapprochement scrotale dans 4 cas et 2 cas de greffe cutanée.

Conclusion: La gangrène de Fournier est une urgence médico-chirurgicale. Son incidence est mal connue dans notre pays (Mali). La recherche précoce des indices de suspicion diagnostic et une prise en charge rapide et efficace permettent de réduire considérablement les complications qui peuvent être fatales.

Conflit d'intérêt : aucun

\section{Référence}

1-P Sarkis, F Farran, R Khoury, G Kamela, E Nemra, J Biajini et al. Gangrène de Fournier : Revue de la littérature récente. Prog Urol 2009 ; $19: 75-84$

2- K Dje, R Lebeau, B Diane, C C Vodi, N Coulibaly, I S Sangaré. La gangrène périnéale à propos de 78 observations. Afr journal of urology 2006 ; 12(1) :44-50.

3- J A Fournier. Gangrène foudroyante de la Verge. Med Prat 1883; 4(5) : 89-97.

4-K Borki, A Ait Ali, A Choho, M Daali, S Alkandry, J L André. La gangrène périnéo-scrotale: A propos de 60 cas. E-mémoires de l'académie nationale de chirurgie $2002 ; 1(4): 49-54$
5-J Hubert, G Fournier, P Mangin, M MPunga. Gangrène des organes génitaux externes. Prog Urol. 1995; 5: 911-924

6-R Hodonou, P PHounnasso, D G Gbessi, C Akpo. Les gangrènes péno-périnéo-scrotales. Aspects épidémiologiques, diagnostiques et thérapeutiques. A Propos de 32 Cas. Prog Urol. 2000; 10: 271-6

7-E Benizri, P Fabiani, G Migliori. Les gangrènes du Périnée. Analyse de 24 observations. Prog Urol. 1992; 2 : 882-91.

8-S. Ettalbi et al. La Gangrène Périnéoscrotale: Profil épidémiologique et aspects thérapeutiques. À propos de 45 cas. Annales de chirurgie plastique esthétique 2013; $58: 310-320$.

9-F Michel, A Fauchery, M Belhadj, J F Couailler, R M Champetier. Apport de l'imagerie dans le diagnostic précoce de la gangrène de fournier. Prog Urol. 1997; 7:471-5

10-D Toutti, A. Ameur, A. Beddouch, H Oukheira. Les gangrènes périnéo-scrotales à propos de 29 cas. Médecine du maghreb 1999;78 : 19-22.

11-A El Moussaoui, R Aboutaieb, A Joual, M El Mrini, F Meziane, S Benjelloun. Les Gangrènes Péno-Périnéo-Scrotales. Analyse de quarante-neuf Cas. Ann. Urol 1994; 28:142-7 